\title{
LEPROSY. WHO WEEKLY EPIDEMIOLOGICAL RECORD, Number 3, (1979), pp. 17-23
}

The above article from a WHO Chronicle of 1977 should be read in close conjunction with detailed information just published by WHO in a Weekly Epidemiological Record of 1979. In fact this refers to the world-wide distribution of leprosy as at 1975, but is nevertheless the most up-to-date and authoritative source of data currently available on the estimated and registered numbers of leprosy cases in virtually all countries where leprosy is endemic. The last two paragraphs of the accompanying text are worth quoting in full:

"Apart from the American Region, where for each country an estimate of the total number of cases accompanies the total of registered cases, less than a third of the countries provided estimates of the total cases. This is to be regretted since the figures which are available suggest that perhaps only one third of the total cases in the world are as yet detected.

It is probable that the total number of cases in 1975 is not substantially different from that of $1968 . "$ 